\title{
Protein Based Drug Discovery
}

\author{
Joshi B. ${ }^{1}$, Gupta G. ${ }^{2}$, Gupta N. ${ }^{3}$, Gupta M. ${ }^{3}$, Trivedi S. ${ }^{4}$, Patil P. ${ }^{6}$, Jhadav A. ${ }^{5}$, Vamsi K.K. ${ }^{7}$, Khairnar Y. \\ Boraste A. ${ }^{5}$, Mujapara A. \\ ${ }^{1}$ Rural College of Pharmacy, D.S Road, Bevanahalli, Banglore \\ ${ }^{2}$ S.D.S.M. College Palghar, Mumbai \\ ${ }^{3}$ Sindhu Mahavidyalaya Panchpaoli Nagpur \\ ${ }^{4}$ V.V.P. Engineering College, Rajkot, Gujrat \\ ${ }^{5}$ Padmashree Dr. D.Y. Patil University, Navi Mumbai, 400614, India \\ ${ }^{6}$ Dr. D. Y. Patil ACS College, Pimpri, Pune \\ ${ }^{7}$ Rai foundations College CBD Belapur Navi Mumbai \\ ${ }^{8}$ Sir PP Institute of Science, Bhavnagar
}

\begin{abstract}
New drug target discovery is currently very popular with a great potential for advancing biomedical research and chemical genomics. Drug discovery is the process of discovering and designing drugs that includes target identification, target validation, lead identification, lead optimization and introduction of the new drugs to the public. G protein-coupled receptors are one of the most important drug targets. In the current scenario of drug research, approximately $60 \%$ of drug target molecules are located at the cell surface, and half of them are GPCRs. Fragment-based drug discovery is established as an alternative approach to high-throughput screening for generating novel small molecule drug candidates. Nanotechnology-based drug delivery systems have seen recent popularity due to their favorable physical, chemical, and biological properties, and great efforts have been made to target nanoDDSs to specific cellular receptors. Protein-protein interactions regulate a wide variety of important cellular pathways, and therefore represent a highly populated class of targets for drug discovery. An analysis of individual proteinprotein interaction systems has recently yielded success in the discovery of drug-like inhibitors.
\end{abstract}

Keyword- Drug discovery, GPCR, Nanotechnology, Protein Crystallography, Protein modeling, Protein structure

\section{Target discovery and validation}

Drug discovery and development approaches rely extensively on the identification and validation of appropriate targets; for example, those with marketable and robust therapeutics. Wide-ranging efforts have been directed at this problem and various approaches have been developed to identify disease-associated genes as candidates. Statistical significance that successful drug targets, in addition to their linkage to disease, share common characteristics those are disease-independent. Marked differences in functional category, sequence variability and tissue specificity are observed between known targets and average proteins. This results lead to an interesting hypothesis: potentially good drug targets shall have some desired properties, which refer to as drug targetlikeness that are beyond their diseaseassociations. The encouraging results will invite future systematic proteomic scale experiments to gather necessary protein characteristics data for the accurate and predictive definition of drug target-likeness, providing a new perspective toward understanding and pursuing effective therapeutics [4]. Sepsis-induced multi-organ failure continues to have a high mortality and the liver is an organ central to the disease pathogenesis. The objective of this study was to identify the liver proteins that change in abundance with sepsis and subsequently identify new drug targets [1]. Mass spectrometric techniques, specifically the analysis of complex peptide mixtures by liquid chromatography and tandem mass spectrometry (shotgun proteomics) has been at the centre of proteomics research for the past decade. To overcome some of the fundamental limitations of the approach, including its limited sensitivity and high degree of redundancy, new proteomic workflows are being developed. Targeting methods in which specific peptides are selectively isolated, identified and quantified are particularly promising. Development of the target-driven research approaches with their ability to detect and quantify identical, non-redundant sets of proteins in multiple repeat analyses will be crucially important for the application of proteomics to biomarker discovery and validation, and to systems biology research [2]. Deregulation of the ubiquitin-proteasome system has been implicated in the pathogenesis of many human diseases, including cancer, neurodegenerative disorders and viral diseases. Recent approval of the proteasome inhibitor bortezomib (Velcade) for the treatment of multiple myeloma and mantle cell lymphoma establishes this system as a valid target for cancer treatment. Ubiquitin specific proteases (USP) are de-ubiquitinating enzymes which remove ubiquitin from specific protein substrates and allow protein salvage from proteasome degradation, regulation of protein localization or activation. Due to their protease activity and their involvement in several pathologies, Ubiquitin specific proteases are emerging as potential target sites for pharmacological interference in the ubiquitin regulatory machinery. We will review here this 
class of enzymes from target validation to small molecule drug discovery [3]. Protein microarrays technology is an emerging class of proteomic technologies, are quickly becoming essential tools for large-scale and high throughput biochemistry and molecular biology. Recent progress has been made in all the key steps of protein microarray fabrication and application, such as the large-scale cloning of expressionready prokaryotic and eukaryotic Open reading frames, high throughput protein purification, surface chemistry, protein delivery systems, and detection methods. Two classes of protein microarrays are currently available: analytical and functional protein microarrays. In the case of analytical protein microarrays, well-characterized molecules with specific activity, such as antibodies, peptide-MHC complexes, or lectins, are used as immobilized probes. Functional protein microarrays are being increasingly applied to many areas of biological discovery, including drug target identification/validation and studies of protein interaction, biochemical activity, and immune responses. Great progress has been achieved in both classes of protein microarrays in terms of sensitivity and specificity, and new protein microarray technologies are continuing to emerge. Protein microarrays have found novel applications in both scientific research and clinical diagnostics [5]. Drug target discovery is a great potential for advancing biomedical research and chemical genomics and innovative strategies have been developed to aid the process of target identification, either by elucidating the primary mechanism-of-action of a drug, by understanding side effects involving unanticipated 'off-target' interactions, or by finding new potential therapeutic value for an established drug. Several promising proteomic methods have been introduced for directly isolating and identifying the protein targets of interest, which are bound by active small molecules or for visualizing enzyme activities affected by drug treatment. Progress has been made in this rapidly advancing field speeding the clinical validation of drug candidates and the discovery of the novel targets for lead compounds developed using cell-based phenotypic screens [6]. Pharmacogenetics is the intersection of the fields of pharmacology and genetics and how genetic variations affect the ways in which people respond to drugs. Pharmacogenomics has the potential to radically change the way health care is provided and it is only in its infancy. In the future research, pharmacogenomics could find uses along the entire drug discovery and development timeline process, all the way from target discovery and validation to late-stage clinical trials. Pharmacogenomics analytical tests could find their way into the doctor's office as a means to get the right medicine to the right patient at the right time. Genetics and genomics are often used synonymously and pharmacogenetics is more focused in scope than and is viewed as a subset of pharmacogenomics, which encompasses factors beyond those that are inherited [7]. Proteomics technologies have produced an abundance of drug targets that is creating a bottleneck in drug development process and is an increasing need for better target validation for new drug development and proteomic technologies are contributing to it. Identifying new potential protein drug target within a cell is a major challenge in modern drug discovery and techniques for screening the proteome are, therefore, an important tool. Major difficulties for target identification include the separate ion of proteins and their detection. Proteomics technology is applicable for protein analysis and bioinformatics based analysis gives the comprehensive molecular description of the actual protein component. Bioinformatics and computational biology is being increasingly used to support target validation by providing functionally predictive information mined from databases and experimental datasets using a variety of computational tools [8]. Drug discovery technology is the process of discovering and designing drugs, which includes target identification, target validation, lead identification, lead optimization and introduction of the new drugs to the public. This methodology is very important, involving analyzing the causes of the diseases and finding ways to tackle them [9]. The strong link between gene expression of mitotic Aurora kinases and cancer has stimulated a very high interest in developing Aurora kinase inhibitors for cancer therapy. Validation of Aurora kinases as targets, and development of pharmacodynamic biomarkers for inhibitors of Aurora kinases, provides an example of how target validation can help the drug discovery process; also, of how to interpret results depending on the technology used. The principal tools, concepts, and strategies of target and biomarker validation for Aurora kinases, with emphasis on validation results derived from RNAinterference experiments. These data were essential for the decision to enter the next steps in drug development and for the selection of the appropriate biomarkers for clinical trials [10]. BK virus large $T$ antigen (LTA) is a hexameric protein with a helicase activity that is powered by ATP hydrolysis. A mutant virus with Lys420Ala, Arg421Ala, and Asp504Ala mutations at the ATP binding sites showed marked reduction in viral fitness. This observation indicates that high throughput screening for ATPase inhibitors will be valid strategy to discover anti-BKV drugs [11]. Tuberculosis disease still remains one of the largest killer infectious diseases, warranting the identification of newer targets and drugs. Identification and validation of appropriate targets 
for designing drugs are critical steps in drug discovery, which are at present major bottlenecks. A majority of drugs in current clinical use for many diseases have been designed without the knowledge of the targets, perhaps because standard methodologies to identify such targets in a high-throughput fashion do not really exist. Now with different kinds of 'omics' data that are now available, computational approaches can be powerful means of obtaining short-lists of possible targets for further experimental validation [12].

\section{GPCRs drug discovery}

G protein-coupled receptors (GPCRs) represent $50-60 \%$ of the current drug targets and no doubt that this family of membrane proteins plays a crucial role in drug discovery today. However, a number of drugs based on GPCRs have been developed for such different indications as cardiovascular, metabolic, neurodegenerative, psychiatric, and oncologic diseases. Owing to the restricted structural information on GPCRs, only limited exploration of structure-based drug design has been possible. Much effort has been dedicated to structural biology on GPCRs and very recently an X-ray structure of the beta2adrenergic receptor was obtained. This breakthrough will certainly increase the efforts in structural biology on GPCRs and furthermore speed up and facilitate the drug discovery process [13]. Understanding the ternary complex between $\mathrm{G}$ protein-coupled receptors (GPCRs), cognate $G$ proteins, and their ligand is an important landmark for drug discovery. Yet, little is known about the specific interactions between GPCRs and G proteins. For a better perspective on the ternary complex dynamics, we adapted a beta (2)-adrenergic receptor(beta(2)AR)tetGs(alpha) reconstitution system and found evidence that for efficient coupling of the beta(2)AR to Gs does not require specific interactions between the betagamma-subunits and the beta(2)AR. The advantages of analysis of $G$ protein activation by using beta (2) $A R$ receptor-tetGs (alpha) system in vitro at the close proximity of the receptor may constitute a simple screening system that avoids false positives and potentially adapted to screen drugs for other GPCRs [14]. G protein-coupled receptors (GPCRs) represent the largest class of targets in drug discovery, one-third of all marketed drugs are active at GPCRs and drugs targeted at GPCRs are marketed in virtually every therapeutic area. G protein-coupled receptors can be classified by virtue of their coupling to second messenger signaling systems. In the last decade functional evaluation of Galphaq-coupled GPCRs has been enabled by advances in fluorescence dye-based methodologies and detection instrumentation. Investigations into the bioluminescence of jelly fish in the early 1960s isolated the photoprotein aequorin that required only the addition of calcium to generate a luminescent signal. New development of sensitive detection platforms with integrated fluidics for liquid handling has revived interest in bioluminescence as an alternative to chemical fluorophore-based detection for characterizing the pharmacology of this target class [15]. Fluorescent dyes are sensitive to changes in intracellular calcium have become increasingly popular in G protein-coupled receptor (GPCR) drug discovery for several reasons. The assays using the dyes are easy to perform and are of low cost compared to other assays. Second, most non-Galpha (q)-coupled GPCRs can be tweaked to modulate intracellular calcium by $\mathrm{co}-$ transfection with promiscuous or chimeric/mutated $\mathrm{G}$ proteins making the calcium assays broadly applicable in GPCR research. Third, the price of instruments capable of measuring fluorescent-based calcium indicators has become significantly less making them obtainable even for academic groups. Measuring the changes in intracellular calcium levels in living mammalian cells based on the fluorescent calcium binding dye, fluo-4 [16]. Functional coupling of heterologous $G$ protein-coupled receptors to the pheromone-response pathway of the budding yeast Saccharomyces cerevisiae is well established as an experimental system for ligand identification and for characterizing receptor pharmacology and signal transduction mechanisms. A number of groups have developed yeast strains using various modifications to this signaling pathway, especially manipulation of the $G$ protein alpha subunit Gpa1p, to facilitate coupling of a wide range of mammalian GPCRs. These systems is the simplicity and low cost of yeast cell culture enabling the assays to be set up rapidly in academic or industrial labs without the requirement for expensive technical equipment. Assays are configured such that agonism of the GPCR leads to induction of a reporter gene and/or growth of the yeast [17]. Drug discovery campaigns for $G$ protein-coupled receptors (GPCRs) heavily rely on assay technologies that use artificial cell systems tailored to a point-ofcontact readout and as a consequence are mostly pathway biased. Recently, developed label-free optical biosensor cellular assays that are capable of examining systems cell biology of endogenous receptors and systems cell pharmacology of GPCR ligand in both physiologically and disease relevant environments [18]. Deorphanization of the large group of $\mathrm{G}$ protein-coupled receptors (GPCRs), which an endogenous activating ligand has not yet been identified (orphan GPCRs) has become increasingly difficult. A specialized technique that has been successfully applied to deorphanize some of these GPCRs involves two-electrode 
voltage-clamp recordings of currents through ion channels that are activated by GPCRs heterologously expressed in Xenopus oocytes. The ion channels that couple to GPCR activation in Xenopus oocytes can be endogenous calciumactivated chloride channels (CaCCs) or heterologously expressed G protein-coupled inwardly rectifying potassium channels (GIRKs). Detection of GPCR activation by recordings of currents through CaCCs that are activated by calcium release from the endoplasmic reticulum and thus the $\mathrm{G}(\mathrm{q})$ signaling pathway [19]. Indole scaffold probably represents one of the very important structural subunits for the discovery of new drug candidates. The demonstration that many alkaloids contain the indole nucleus, the recognition of the importance of essential amino acid tryptophan in human nutrition and the discovery of plant hormones served to bring about a massive search on indole chemistry, giving rise to a vast number of biologically active natural and synthetic products, with a wide range of therapeutic targets, such as antiinflammatories, phosphodiesterase inhibitors, 5hydroxytryptamine receptor agonists and antagonists, cannabinoid receptors agonists and HMG-CoA reductase inhibitors. Many of these target-receptors belong to the class of GPCRs (integral membrane G-protein coupled receptors) and possess a conserved binding pocket that is recognized by the indole scaffold in a common complementary binding domain, explaining the great number of drugs that contain the indole substructure, such as indomethacin, ergotamine, frovatriptan, ondansetron, tadalafil, among many others [20]. Drug discovery efforts advance in step with advancements in assay technologies, as new technologies provide new lenses through which biology can be viewed. The novel information gathered results in the better understanding of drug-target interactions leading to better decision making during the drug discovery process. Rapid development is within label-free technologies. Label-free technologies offer many distinct advantages to the drug discovery workflow. Such novel new technology is the CellKey System, an impedance-based label-free live cell assay platform. The system is based on impedance technology and is a universal platform for the functional measurement of all classes of G-protein coupled receptors (GPCRs) [21]. Recent advances in structural biology for G-protein-coupled receptors (GPCRs) have provided new opportunities to improve the definition of the transmembrane binding pocket. A reference set of 44 residue positions accessible for ligand binding was defined through detailed analysis of all currently available crystal structures. This was used to characterize pharmacological relationships of Family A/Rhodopsin family GPCRs, minimizing evolutionary influence from parts of the receptor that do not generally affect ligand binding. The transmembrane binding site reference set, particularly when coupled with a means of identifying the subset of ligand binding residues, provides a general paradigm for understanding the pharmacology/selectivity profile of ligand at Family A GPCRs. This has wide applicability to GPCR drug design problems across many disease areas [22]. G protein-coupled receptors (GPCRs) are the target of approximately $40 \%$ of all approved drugs and continue to represent a significant portion of drug discovery portfolios across the pharmaceutical industry. Ligandbinding assays were used to identify compounds that targeted GPCRs. GPCR drug discovery efforts have moved toward the utilization of functional cell-based assays for HTS. Many of these assays monitor the accumulation of a second messenger such as CAMP or calcium in response to GPCR activation. Calcium stores are released from the endoplasmic reticulum when Galphaq-coupled GPCRs are activated. Although Galphai- and Galphas-coupled receptors do not normally result in this mobilization of intracellular calcium, they can often be engineered to do so by expressing a promiscuous or a chimeric Galphaprotein that couples to the calcium pathway. Thus calcium mobilization is a readout that can theoretically be used to assess activation of all GPCRs [23]. Human G-protein coupled receptors (hGPCRs) comprise the most prominent family of validated drug targets. More than $50 \%$ of approved drugs reveal their therapeutic effects by targeting this family. Accurate models would greatly facilitate the process of drug discovery and development. Also, 3-D structure prediction of GPCRs remains a challenge due to limited availability of resolved structure. The X-ray structures have been solved for only four such proteins. The identity between hGPCRs and the potential templates is mostly less than $30 \%$, well below the level at which sequence alignment can be done regularly. In this study, we analyze a large database of human G-protein coupled receptors that are members of family $A$ in order to optimize usage of the available crystal structures for molecular modeling of hGPCRs [24]. Allosteric receptor ligand bind to a recognition site that is distinct from the binding site of the endogenous messenger molecule. As a consequence, allosteric agents may attach to receptors that are already transmitter-bound. Ternary complex formation opens an avenue to qualitatively new drug actions at $G$ protein-coupled receptors (GPCRs), in particular receptor subtype selective potentiation of endogenous transmitter action. Consequently, suitable exploitation of allosteric recognition sites as alternative molecular targets could pave the way to a drug discovery paradigm different from those aimed at mimicking or blocking the effects of endogenous (orthosteric) 
receptor activators. The number of allosteric ligand reported to modulate GPCR function is steadily increasing and some have already reached routine clinical use [25].

\section{Protein structure}

Rett syndrome (RTT) is a severe postnatal neurological disorder caused by mutations in the methyl-CpG binding protein 2 (MECP2) genes and deficit in bioaminergic metabolism has been identified at the cellular and molecular levels, in more than 200 patients. Recently available transgenic mouse strains with a defective Mecp2 gene also show abnormalities, strongly suggesting that there is a direct link between the function of the MECP2 protein and the metabolism of biogenic amines. Biogenic amines appear to have an important role in the pathophysiology of Rett syndrome, for several reasons. Biogenic amines modulate a large number of autonomic and cognitive functions. Many of these functions are affected in RTT patients. Biogenic amines are the only neurotransmitters that have repeatedly been found to be altered in RTT patients [26]. Stealth nanoparticles are generally obtained after modifying their surface with hydrophilic polymers, such as PEG. In this study, the effect of a phospholipid (DG) or protein (BSA) inclusion in porous cationic polysaccharide (NP $(+))$ on their physico-chemical structure and the effect on complement activation was analyzed [27]. Unfolded protein response (UPR) has evolved to counter the stresses that occur in the endoplasmic reticulum (ER) as a result of misfolded proteins. This sophisticated quality control system attempts to restore homeostasis through the action of a number of different pathways that are coordinated in the first instance by the ER stress-senor proteins IRE1, ATF6 and PERK. Prolonged ER-stress-related UPR can have detrimental effects on cell function in the longer term, may induce apoptosis. Connective tissue cells such as fibroblasts, osteoblasts and chondrocytes synthesise and secrete large quantities of proteins and mutations in many of these gene products give rise to heritable disorders of connective tissues. Until recently, these mutant gene products were thought to exert their effect through the assembly of a defective extracellular matrix that ultimately disrupted tissue structure and function. It is now clear that ER stress and UPR, because of the expression of a mutant gene product, is not only a feature of, but may be a key mediator in the initiation and progression of a whole range of different connective tissue diseases [28]. Monoclonal antibody (MAb) (designated as 15E2) against the IBV M protein was prepared and a series of 14 partially-overlapping fragments of the IBV M gene were expressed with a GST tag. These peptides were subjected to enzyme-linked immunosorbent assay (ELISA) and western blotting analysis using MAb 15E2 to identify the epitope. Linear motif, FATFVYAK, which was located at the $\mathrm{C}$-terminus of the $\mathrm{M}$ protein, was identified by MAb 15E2. 15E2 showed reactivity with the FATFVYAK motif, expressed as a GST fusion protein, in both western blotting and in an ELISA, this motif represented a linear B-cell epitope of the M protein. The FATFVYAK motif was the minimal requirement for reactivity as demonstrated by analysis of the reactivity of 15E2 with several truncated peptides that were derived from the motif. Alignment and comparison of the 15E2-defined epitope sequence with the sequences of other coronaviruses indicated that the epitope is well conserved among chicken and turkey coronaviruses. Identified epitope should be useful in clinical applications and as a tool for the further study of the structure and function of the $M$ protein of IBV [29]. The group of proteins, which contain a thioredoxin (Trx) fold, is huge and diverse. Assessment of the variation in catalytic machinery of Trx fold proteins is essential in providing a foundation for understanding their functional diversity and predicting the function of the many uncharacterized members of the class. The proteins of the Trx fold class retain common features-including variations on a dithiol $\mathrm{CxxC}$ active site motif-that lead to delivery of function. Domain structure in the fold class is varied and modular, with $2.8 \%$ of sequences containing more than one Trx fold domain. Most member proteins are bacterial. The fold class exhibits many modifications to the $\mathrm{CxxC}$ active site motifonly $56.8 \%$ of proteins have both cysteines, and no functional groupings have absolute conservation of the expected catalytic motif. Only a small fraction of Trx fold sequences have been functionally characterized. Unifying context provided by work can guide the comparison of members of different Trx fold superfamilies to gain insight about their structure-function relationships, illustrated here with the thioredoxins and peroxiredoxins [30]. Proteomic analysis occupies an increasingly important place in gamete and embryo biology as an independent tool of discovery and as a means of follow-up to transcriptional profiling. Proteomics have been and will be increasingly helpful in many areas of reproductive biology, including applied science and technology development. Areas likely to be impacted most rapidly by proteomic knowledge include fertility evaluation in male farm animals, male infertility diagnostics in humans, assessment and optimization of oocyte and embryo culture protocols, selection of fittest oocytes for assisted fertilization and selection of most competent embryos for embryo transfer. Oocyte proteomics will help us understand the process of oogenesis and oocyte maturation, and to discover non-invasive markers of oocyte 
quality. Sperm proteomics correlate with normal sperm structure and function and can be applied to discover novel biomarkers of farm animal fertility and diagnostic markers of human male infertility [31]. Availability of genome sequences, and inferred protein coding genes, has led to several proteome-wide studies of isoelectric points. Isoelectric points are distributed following variations on a biomodal theme that originates from the predominant acid and base amino acid sidechain pKas. Relative populations of the peaks in such distributions may correlate with environment, either for a whole organism or for subcellular compartments. There is also a tendency for isoelectric points averaged over a subcellular location to not coincide with the local $\mathrm{pH}$, which could be related to solubility. The correlation of other $\mathrm{pH}$-dependent properties, calculated from 3D structure, with subcellular $\mathrm{pH}$ [32]. Molecular aspects of thermal adaptation of proteins are studied by following the co-evolution of temperature dependence, conformational stability and substrate specificity in a cold-active lipase modified via directed evolution. Temperature dependence and selectivity turned out to be mutually dependent. While the wild type protein was strictly specific for short-chain triglycerides (C4) in the temperature range 10 degrees $\mathrm{C}-50$ degrees $\mathrm{C}$ and displayed highest activity in the cold, its stabilized variant was able to accept C8 and C12 molecules and its selectivity was temperature dependent. Strong evidence for local stabilization effects in the active site region provided by two independent approaches. Differential scanning fluorimetry revealed that the exposure of hydrophobic patches (as the active site is) precedes denaturation and molecular dynamics simulations confirmed that stability was obtained by restriction of the mobility of the lid, a flexible structure that regulates the access to the enzyme active site and influences its stability [33].

Modeling and fragment based drug discovery Discovery of novel inhibitors of prostaglandin D2 synthase (PGDS) through fragment-based lead generation and structure-based drug design is describe here. Library of 2500 low-molecularweight compounds was screened using $2 \mathrm{D}$ nuclear magnetic resonance (NMR), leading to the identification of 24 primary hits. Structure determination of protein-ligand complexes with the hits enabled a hit optimization process, whereby harvested increasingly more potent inhibitors out of our corporate compound collection. Six novel high-resolution PGDS complex structures were determined, and 300 hit analogues were tested. The best inhibitor (IC 50 $=21 \mathrm{nM}$ ) is one of the most potent inhibitors of PGDS to date. It may enable new functional in vivo studies of PGDS and the prostaglandin metabolism pathway [34]. Recent research developments in fragment-based drug-discovery methods have emphasis on informatics and modeling requirements. Fragment-based methods have become established as a powerful approach in structure-based lead discovery. A number of successful projects have been announced recently, where fragments have had a central role in hit generation and lead optimization, leading to candidates being considered for clinical trials. There are still many opportunities for new development, such as improving the structural diversity of fragment libraries, strategies for fragment evolution, and methods for predicting fragment binding modes [35]. Discovery of pharmaceutical agents is a complex, lengthy and costly process, critically depending on the availability of rapid and efficient screening methods. When targets are large, multidomain proteins, their complexity may affect unfavorably technical feasibility, costs and unambiguity of binding test interpretation. Possible strategy to overcome these problems relies on molecular design of receptor fragments that are: sensible targets for ligand screenings, conformationally stable also as standalone domains, easily synthesized and immobilized on chip for Biacore experiments [36]. Detection of outliers is a complex and challenging area of research in chemical theory. Among current notions, that of outliers in the chemical space-descriptors--is meaningful with multiple applications in the field of drug discovery and predictive modeling. Recursive method is developed, which quantifies the contribution of fragments to compound description and identifies outliers in chemical structure databases according to a novel definition. In apposite to existing detection routes, this approach avoids the use of thresholds usually required to quantify outlying behavior. Three chemical databases are investigated to demonstrate its generality and flexibility. The result shows, a new species of outliers, compounds with no specific structural features, rather than unique ones [37]. The worldwide spread of $\mathrm{A} / \mathrm{H} 5 \mathrm{~N} 1$ avian influenza with high virulence has highlighted the potential threat of human influenza pandemic. Tamiflu and Relenza are currently the only two anti-influenza drugs targeting the neuraminidase (NA) enzyme of human influenza virus. Reports of the emergence of drug resistance further make the development of new potent anti-influenza inhibitors a priority. X-ray crystallographic study of $\mathrm{A} / \mathrm{H} 5 \mathrm{~N} 1$ avian influenza NA subtypes has demonstrated that there exist two genetically distinct groups, group-1 (N1, N4, N5 and N8) and group-2 (N2, N3, N6, N7 and N9), whose conformations are substantially different. A threedimensional structure of $\mathrm{N} 1$ subtype of human influenza type $A$ virus $(\mathrm{N} 1 \mathrm{hA})$ has been generated by homology modeling using the $\mathrm{X}$-ray crystallographic structure of $\mathrm{N} 1$ subtype of avian 
influenza virus (N1aA) as the template [38]. Model describing the structure and conformational preferences of the HIV-Haiti V3 loop in the geometric spaces of Cartesian coordinates and dihedral angles was generated in terms of NMR spectroscopy data published in literature. Conservative structural motif embracing one of the potential sites of the gp120 $\mathrm{N}$-linked glycosylation was detected, which seems to be a promising target for the HIV-1 drug design. The implications are discussed in conjunction with the literature data on the biological activity of the individual amino acids for the HIV-1 gp120 V3 loop [39]. Fab-fragments of the monoclonal antibody 6B4, raised against human glycoprotein Ibalpha (GPlbalpha), have a powerful antithrombotic effect in baboons by blocking the GPIbalpha binding site for von Willebrand factor (VWF), without significant prolongation of the skin bleeding time. Injection of doses of 0.1 to $1.5 \mathrm{mg} / \mathrm{kg}$ of h6B4-Fab in baboons showed that both pharmacokinetics and ex-vivo bio-activity of the molecule were to a large extent preserved [40]. In Bayesian approach, prior knowledge and uncertainty of the mathematical model are incorporated into the estimated model and its parameters. Molecular interaction data are strengthened by narrowing the atom classification to 14 atom types, focusing on independent molecular contacts, which lie within a short cutoff distance, and symmetrizing the interaction data for the molecular fragments. The fact that an atom will simultaneously interact with several molecular fragments forming a cohesive network of interactions is exploited by introducing two strategies that combine the predictions of atom types given by multiple fragments. The accuracy of these combined predictions is compared with those based on an individual fragment. Exhaustive validation analyses and qualitative examples (e.g., the ligand-binding domain of glutamate receptors) demonstrate that these improvements lead to effective modeling and prediction of molecular interactions [41].

\section{Protein crystallography in drug discovery}

Modification of left-hand side (LHS) of biphenyl analogues containing an acylsulfonamide moiety in the development of potent and selective human beta (3)-adrenergic receptor (AR) agonists was investigated [42]. Novel proapoptotic Smac mimics/IAPs inhibitors have been designed synthesized and characterized. Computational models and structural studies (crystallography, NMR) have elucidated the SAR of this class of inhibitors, and have permitted further optimization of their properties. In vitro characterization (XIAP BIR3 and linker-BIR2BIR3 binding, cytotox assays, early ADMET profiling) of the compounds has been performed, identifying one lead for further in vitro and in vivo evaluation [43]. The X-ray crystal structure of an amide-based progesterone receptor (PR) partial agonist bound to the PR ligand binding domain, a novel PR partial agonist class containing a pyrrolidine ring was designed. Members of this class of $\mathrm{N}$-alkylpyrrolidines demonstrate potent and highly selective partial agonism of the progesterone receptor, and one of these analogs was shown to be efficacious upon oral dosing in the OVX rat model of estrogen opposition [44]. Catechol-O-methyltransferase is responsible for metabolism of catechol neurotransmitter and xenobiotics. Analysis of the structural differences among the previously reported inhibitor complexes, coumarine-based inhibitor (4-phenyl7, 8-dihydroxycoumarine: 4PCM) bound structure provides the explanation for inhibitor binding and can be used for future inhibitor design [45]. Glutamine:fructose-6-phosphate

amidotransferase (GFAT) is a rate-limiting enzyme in the hexoamine biosynthetic pathway and plays an important role in type 2 diabetes. The diversity of the CF helix near the active site suggests the helix is a major target for drug design [46]. Protein-tyrosine phosphatases (PTPs) are considered important therapeutic targets because of their pivotal role as regulators of signal transduction and thus their implication in several human diseases such as diabetes, cancer, and autoimmunity. PTP1B has been the focus of many academic and industrial laboratories because it was found to be an important negative regulator of insulin and leptin signaling, and hence a potential therapeutic target in diabetes and obesity [47]. Surface transmembrane glycoprotein is responsible for mediating virion attachment to cell and subsequent virus-cell membrane fusion. Crystal structure of the fusion core of mouse hepatitis virus $S$ protein, which represents the first fusion core structure of any coronavirus, reveals a central hydrophobic coiled coil trimer surrounded by three helices in an oblique, antiparallel manner. Drug discovery strategies aimed at inhibiting viral entry by blocking hairpin formation may be applied to the inhibition of a number of emerging infectious diseases, including severe acute respiratory syndrome [48]. Aminoimidazole-4-carboxamide ribonucleotide transformylase (AICAR Tfase), one of the two folate-dependent enzymes in the de novo purine biosynthesis pathway, is a promising target for anti-neoplastic chemotherapy. The crystal structure of AICAR Tfase with 326203-A at 1.8, a resolution revealed a unique binding mode compared with antifolate inhibitors. The sulfonate group of 326203-A appears to form the dominant interaction of the inhibitor with the proposed oxyanion hole through interaction with a helix dipole and Lys(267) [49]. Inhibition of cyclin Aand cyclin E-associated cyclin-dependent kinase2 (CDK2) activities is an effective way of 
selective induction of apoptotic cell death via the E2F pathway in tumour cells. The cyclin groove recognition motif (CRM) in the natural CDKinhibitory (CDKI) tumour suppressor protein p27KIP1 was used as the basis for the design and synthesis of a series of cyclic peptides whose biological activity and structural characterisation by NMR and X-ray crystallography is reported. The structural basis for the potency increase in cyclic versus linear peptides was demonstrated through the determination and interpretation of X-ray crystal structures of complexes between CDK2/cylin A (CDK2A) and a constrained pentapeptide [50].

\section{Protein drug delivery system and nanotechnology}

Nanotechnologies hold considerable promise of advances in many sectors especially the biomedical fields, since the materials used are of the appropriate dimensions to interact with important biological matter such as proteins, DNA and viruses. The use of nanotechnologies will probably be second in importance only to biotechnologies [51]. Nanoparticles have unique physicochemical properties which make them promising platforms for drug delivery. Also, immune cells in the bloodstream (such as monocytes, platelets, leukocytes, and dendritic cells) and in tissues (such as resident phagocytes) have a propensity to engulf and eliminate certain nanoparticles. A nanoparticle's interaction with plasma proteins (opsonins) and blood components (via hemolysis, thrombogenicity and complement activation) may influence uptake and clearance and hence potentially affect distribution and delivery to the intended target sites [52]. Nanotechnology can play a pivotal role by delivering drugs in a targeted fashion to the malignant cells that will reduce the systemic toxicity of the anticancer drug. The developments of a nanoparticle-based targeted delivery system for in vitro and in vivo have therapeutic application in pancreatic cancer. In vivo therapeutic efficacy of the targeted delivery system was shown [53]. Nanoconjugates are emerging as promising drug-delivery vehicles because of their multimodular structure enabling them to actively target discrete cells, pass through biological barriers and simultaneously carry multiple drugs of various chemical nature. Nanoconjugates have matured from simple devices to multifunctional, biodegradable, nontoxic and nonimmunogenic constructs, capable of delivering synergistically functioning drugs in vivo [54]. Nanomaterials such as block copolymeric membranes provide a platform for both cellular interrogation and biological mimicry. Their biomimetic properties are based upon the innate possession of hydrophilic and hydrophobic units that enable their integration with a broad range of therapeutic materials. As such, they can be engineered for specific applications in nanomedicine, including controlled/localized drug delivery. PolyDex may be utilized as a localized, highly efficient drug-copolymer composite for active therapeutic delivery to confer antiinflammatory protection or as a platform material for broad drug elution capabilities [55]. Tumorspecific targeting using achievements of nanotechnology is a mainstay of increasing efficacy of anti-tumor drugs. To improve drug targeting covalently conjugation of two different monoclonal antibodies, an anti-mouse transferrin receptor antibody and a mouse autoimmune antinucleosome antibody $2 \mathrm{C} 5$, onto the drug delivery nanoplatform, poly(beta-L-malic acid). Drug delivery system was targeted across mouse endothelial system by the anti-mouse transferring receptor antibody and to the tumor cell surface by the anti-nucleosome antibody 2C5 [56]. Nanotechnology-based drug delivery systems is popular and their favorable physical, chemical, and biological properties, and great efforts have been made to target nanoDDSs to specific cellular receptors. CD44/chondroitin sulfate proteoglycan (CSPG) is among the receptors overexpressed in metastatic melanoma, and the sequence to which it binds within the type IV collagen triple-helix has been identified [57]. Colloidal microgels have recently received attention as environmentally responsive systems and now are increasingly used in applications as carriers for therapeutic drugs and diagnostic agents. Synthetic microgels consist of a crosslinked polymer network that provides a depot for loaded drugs, protection against environmental hazards and template for postsynthetic modification or vectorization of the drug carriers [58]. The concept of nanoscale devices has led to the development of biodegradable selfassembled nanoparticles, which are being engineered for the targeted delivery of anticancer drugs and imaging contrast agents. Nanoconstructs such as these should serve as customizable, targeted drug delivery vehicles capable of ferrying large doses of chemotherapeutic agents or therapeutic genes into malignant cells while sparing healthy cells [59].

\section{Design drugs targeting protein-protein interaction}

Protein-protein interactions are crucial to biological functions. Consequently, designing drugs to control protein-protein interactions is receiving increasing attention. Protein structures can associate in different ways. Analysis of the structures of protein-protein complexes using amino acid sequence order-independent multiple structural comparison algorithms, led us to conclude that the amino acids Trp, Met, and Phe are important for protein-protein interactions [60]. Protein-protein interactions sequester enzymes 
close to their substrates. Protein kinase $C$ (PKC) is one example of a ubiquitous signaling molecule with effects that are dependent upon localization. Short peptides derived from interaction sites between each PKC isozyme and its receptor for activated $C$ kinase act as highly specific inhibitors and have become available as selective drugs in basic research and animal models of human diseases, such as myocardial infarction and hyperglycemia [61]. Modulation of intracellular protein-protein interactions has been and remains a challenging goal for the discovery and development of small-molecule therapeutic agents. New research progress in the pharmacological targeting and understanding at the molecular level of one such interaction that is relevant to cancer drug research, viz. that between the tumour suppressor protein p53 and its negative regulator HDM2 [62]. Protein-protein interactions (PPIs) have an important role in many biological processes suggesting that targeting macromolecular complexes will open new avenues for the design of the next generation of therapeutics. Virtual ligand screening, protein-protein docking, structural predictions and druggable pocket predictions have become established techniques for hit discovery and optimization [63]. For anticancer therapy, stimulation of proapoptotic mitochondrial events in tumor cells and their suppression in surrounding normal cells represents a promising paradigm for new therapies. Mitochondriatargeted disruptors and inhibitors of cyt $\mathrm{c} / \mathrm{CL}$ peroxidase complexes and suppression of $\mathrm{CL}$ peroxidation represent new strategies in antiapoptotic drug discovery [64]. Organic anion transporters (OATs, SLC22) interact with a remarkably diverse array of endogenous and exogenous organic anions. However, little is known about the structural features that determine their substrate selectivity. The substrate binding preferences and transport function of olfactory organic anion transporter, Oat6, in comparison with the more broadly expressed transporter, Oat1 [65]. Long-term exposure to antivirals is associated with serious cellular toxicity to the kidney and other tissues. Organic anion transporters (OATs) are believed to mediate the cellular uptake. Hence cytotoxicity, of many antivirals and antiviral specificity of SLC22a6 and SLC22a8 is consistent with the Xenopus oocyte data. The combined observations suggest SLC22a8 is the major transporter interacting with $\mathrm{ddC}$ and ddl. Quantitative structure-activity relationship analysis of the nine antivirals' physicochemical descriptors with their OAT affinity indicates that antiviral preferences of mOat 1 are explained by high polar surface areas (e.g., phosphate groups); whereas mOat3 prefers hydrogen bond acceptors (e.g., amines, ketones) and low rotatable bond numbers [66]. Most orally bioavailable drugs on the market are competitive inhibitors of catalytic sites, but a significant number of targets remain undrugged, because their molecular functions are believed to be inaccessible to drug-like molecules. This observation specifically applies to the development of small-molecule inhibitors of macromolecular interactions such as proteinmembrane interactions, which have been essentially neglected thus far. Many proteins containing a membrane-targeting domain play a crucial role in health and disease and the inhibition of such interactions therefore represents a very promising therapeutic validation strategy [67]. Modulation of intracellular protein-protein interactions has been and remains a challenging goal for the discovery and development of small-molecule therapeutic agents. Progress in the pharmacological targeting and understanding at the molecular level is relevant to cancer drug research, viz. which between the tumour suppressor protein p53 and its negative regulator HDM2 is reviewed. There is still some way to go as far as optimisation and preclinical development of such leads is concerned, but it is clear already now that antagonists of the p53-HDM2 protein-protein interaction have a good chance of ultimately being successful in providing a new anti-cancer therapy modality, both in monotherapy and to potentiate the effectiveness of existing chemotherapies [68]. Reverse transcriptase (RT) of all retroviruses is required for synthesis of the viral DNA genome. The human immunodeficiency virus type 1 (HIV-1) RT exists as a heterodimer made up of $51-\mathrm{kDa}$ and $66-\mathrm{kDa}$ subunits. The crystal structure and in vitro biochemical analyses indicate that the p66 subunit of RT is primarily responsible for the enzyme's polymerase and RNase $\mathrm{H}$ activities. Both the p51 and p66 subunits are generated from the same coding region, as part of the Pr160(Gag-Pol) precursor protein, there are inherent limitations for studying subunit-specific function with intact provirus in a virologically relevant context [69]. Drug transporters molecules are expressed in many tissues such as the intestine, liver, kidney, and brain, and play key roles in drug absorption, distribution, and excretion. The information on the functional characteristics of drug transporters provides important information to allow improvements in drug delivery or drug design by targeting specific transporter proteins [70].

\section{New peptides drug discovery}

Ever-increasing number of drug-resistant bacteria is a major challenge in healthcare and creates an urgent need for novel compounds for treatment. Host defense antimicrobial peptides have high potential to become the new generation of antibiotic compounds. Antimicrobial peptides 
constitute a major part of the innate defense system in all life forms [71]. HER2/neu and MUC1-based synthetic peptides are prepared and evaluated in an effort to develop peptidebased radiopharmaceuticals derived from tumorassociated-antigens for the detection of breast cancer. The receptors for HER2/neu and MUC1 are overexpressed in various human cancers, breast and ovarian cancer. Relatively low expression of these antigens on normal tissues makes them attractive targets for tumor imaging research [72]. Staphylococcus aureus has one essential type I signal peptidase (SPase), SpsB, which has emerged as a potential target in the search for antibiotics with a new mode of action. Truncated derivative of SpsB, which was nine amino acids longer at the $\mathrm{N}$-terminus compared to the self-cleavage product, retained activity. The specificity constants $(\mathrm{k}(\mathrm{cat}) / \mathrm{K}(\mathrm{m}))$ of the fulllength and the truncated derivative were $1.85+/$ $0.13 \times 10(3) \mathrm{m}(-1) . \mathrm{s}(-1)$ and $59.4+/-6.4 \mathrm{~m}(-1) . \mathrm{s}(-$ $1)$, respectively, as determined using the fluorogenic synthetic peptide substrate [73]. Peptides did not share a common sequence motif, length, or net charge; nonetheless, they shared a mechanism of action that is similar to the natural membrane permeabilizing antimicrobial peptides (AMP). To characterize the selected peptides and to compare the activity of AMPs in vivo and in vitro and report was presented on the biological activity of the same selected peptides in bacteria, fungi, and mammalian cells. Screening of peptide libraries in vitro for members with the appropriate interfacial activity can enable the design, selection, and discovery of novel, potent, and broad-spectrum membrane-active antibiotics [74]. Ternatin, a highly $\mathrm{N}$-methylated cyclic peptide, inhibits fat accumulation in 3T3-L1 cells and reduces fat mass in mice. Ternatin affects the mid-to late differentiation stages of adipocytes. Ternatin also inhibited triglyceride synthesis in rat primary hepatocytes, suggesting that the potential action sites for ternatin are shared by adipocytes and liver [75]. Short amidated neuropeptides are widespread have important functions within the nervous systems of all flatworms (phylum Platyhelminthes) examined, and could therefore represent a starting point for new lead drug compounds with which to combat parasitic helminth infections [76]. Protein transduction domain (PTD)-peptides greatly facilitate the delivery of high molecular weight macromolecules across the blood-brain barrier (BBB). BBB-transport function is highly desirable and helps to enable the development of new therapeutics for treatment of brain disorders. However, the drug discovery process is limited by the generation of a simple and reliable $\mathrm{BBB}$ model that is amenable to testing of large number of samples and simultaneously, reproduces the physiological and functional characteristics of the human BBB [77]. Novel research strategy for the ribosomal synthesis of peptides featuring Cterminal lactam, thiolactone, and alkylamide units is described. The method is based on the concept of genetic code reprogramming involving the flexizymes (flexible tRNA acylation ribozymes) and the PURE (peptide synthesis using recombinant elements) system, in which vacant codons are reassigned to nonproteinogenic amino acids; this enabled us to convert the $\mathrm{C}$ termini of peptides into the above functionalities [78]. CD-NP is a novel chimeric natriuretic peptide (NP) consisting of the 22amino-acid $(A A)$ human $C$-type natriuretic peptide (CNP), a venodilating peptide with limited renal actions and minimal effects on blood pressure, and the 15-AA C-terminus of Dendroaspis NP (DNP). The rationale for the design of CD-NP was to enhance the renal actions of CNP, the ligand for natriuretic peptide receptor- $\mathrm{B}$, but without inducing excessive hypotension [79]. Controlling apoptotic pathways with peptides is showing increasing promise as a strategy in drug development. Peptides are essential tools for drug discovery, as well as preclinical and pharmaceutical drug development [80].

\section{Conclusion}

Applying proteomics to a clinically relevant mouse model of sepsis identified a number of novel proteins that changed in abundance. Application of proteomics techniques into sepsis research can facilitate the discovery of new therapeutic targets. Combining bioinformatics with drug discovery is a very promising method although it faces many problems and pipeline developed provides rational schema for drug target identification that are likely to have high rates of success, which is expected to save enormous amounts of money, resources and time in the drug discovery process. Nanoparticles are able to absorb large amounts of phospholipids or proteins without change in their size or zeta potential. Complement studies showed, which stealth behaviour is observed when they are loaded and saturated either with anionic phospholipid or proteins. A constraint on protein $\mathrm{pH}$-dependence, in relation to the local $\mathrm{pH}$, that is manifested in the $\mathrm{pKa}$ distribution of histidine sub-proteomes.

\section{References}

[1] Dear J.W., Leelahavanichkul A., Aponte A., Hu X., Constant S.L., Hewitt S.M., Yuen P.S., Star R.A. (2007) Crit Care Med. 35(10):2319-28.

[2] Malmstrom J., Lee H., Aebersold R. (2007) Curr Opin Biotechnol. 18(4):378-84.

[3] Daviet L., Colland F. (2008) Biochimie. 90(2):270-83. 
[4] Xu H., Lin M., Wang W., Li Z., Huang J., Chen Y., Chen X. (2007) Proteomics. 7(23):4255-63.

[5] Tao S.C., Chen C.S., Zhu H. (2007) Comb Chem High Throughput Screen. 10(8):706-18.

[6] Sleno L., Emili A. (2008) Curr Opin Chem Biol. 12(1):46-54.

[7] Gomase V.S., Tagore S., Changbhale S.S., Kale K.V. (2008) Curr Drug Metab. 9(3):207-12.

[8] Gomase V.S., Kale K.V., Tagore S., Hatture S.R. (2008) Curr Drug Metab. 9(3):21320.

[9] Chen Y.P., Chen F. (2008) Expert Opin Ther Targets. 12(4):383-9.

[10] Colombo R., Moll J. (2008) Mol Diagn Ther. 12(2):71-6.

[11] Zeng G., Bueno M., Camachos C.J., Ramaswami B., Luo C., Randhawa P. (2009) Antiviral Res. 81(2):184-7.

[12] Raman K., Yeturu K., Chandra N. (2008) BMC Syst Biol. 2:109.

[13] Lundstrom K. (2009) Methods Mol Biol. 552:51-66.

[14] Ratnala V.R., Kobilka B. (2009) Methods Mol Biol. 552:67-77.

[15] Brough S.J., Shah P. (2009) Methods Mol Biol. 552:181-98.

[16] Hansen K.B., Brauner-Osborne H. (2009) Methods Mol Biol. 552:269-78.

[17] Dowell S.J., Brown A.J. (2009) Methods Mol Biol. 552:213-29.

[18] Fang Y., Ferrie A.M., Tran E. (2009) Methods Mol Biol. 552:239-52.

[19] Hansen K.B., Brauner-Osborne H. (2009) Methods Mol Biol. 552:343-57.

[20] De Sa Alves F.R., Barreiro E.J., Fraga C.A. (2009) Mini Rev Med Chem. 9(7):78293.

[21] McGuiness R.P., Proctor J.M., Gallant D.L., van Staden C.J., Ly J.T., Tang F.L., Lee P.H. (2009) Comb Chem High Throughput Screen. 12(8):812-23.

[22] Gloriam D.E., Foord S.M., Blaney F.E., Garland S.L. (2009) J Med Chem. 52(14):4429-42.

[23] Emkey R., Rankl N.B. (2009) Methods Mol Biol. 565:145-58.

[24] Rayan A. (2009) J Mol Model.

[25] De Amici M., Dallanoce C., Holzgrabe U., Trankle C., Mohr K. (2009) Med Res Rev.

[26] Roux J.C., Villard L. (2009) Behav Genet.

[27] Paillard A., Passirani C., Saulnier P., Kroubi M., Garcion E., Benoit J.P., Betbeder D. (2009) Pharm Res.

[28] Boot-Handford R.P., Briggs M.D. (2009) Cell Tissue Res.

[29] Xing J, Liu S, Han Z, Shao Y, Li H, Kong X (2009) J Microbiol. 47(5):589-99.
[30] Atkinson H.J., Babbitt P.C. (2009) PLoS Comput Biol. 5(10):e1000541.

[31] Sutovsky P. (2009) Soc Reprod Fertil Suppl. 66:103-16.

[32] Chan P., Warwicker J. (2009) BMC Biol. 7(1):69.

[33] Gatti-Lafranconi P., Natalello A., Rehm S., Doglia S.M., Pleiss J., Lotti M. (2009) J Mol Biol. 19.

[34] Hohwy M., Spadola L., Lundquist B., Hawtin P., Dahmen J., Groth-Clausen I., Nilsson E., Persdotter S., von Wachenfeldt K., Folmer R.H., Edman K. (2008) J Med Chem. 51(7):2178-86.

[35] Hubbard R.E., Chen I., Davis B. (2007) Curr Opin Drug Discov Devel. 10(3):289-97.

[36] Monfregola L., Vitale R.M., Amodeo P., De Luca S. (2009) Bioorg Med Chem. 17(19):7015-20.

[37] Casalegno M., Sello G., Benfenati E. (2008) $J$ Chem Inf Model. 48(8):1592-601.

[38] Zhang Q., Yang J., Liang K., Feng L., Li S., Wan J., Xu X., Yang G., Liu D., Yang S. (2008) J Chem Inf Model. 48(9):180212.

[39] Andrianov A.M., Veresov V.G. (2007) J Biomol Struct Dyn. 24(6):597-608.

[40] Fontayne A., Vanhoorelbeke K., Pareyn I., Van Rompaey I., Meiring M., Lamprecht S., Roodt J., Desmet J., Deckmyn H. (2006) Thromb Haemost. 96(5):671-84.

[41] Rantanen V.V., Gyllenberg M., Koski T., Johnson M.S. (2003) J Comput Aided Mol Des. 17(7):435-61.

[42] Hattori K., Orita M., Toda S., Imanishi M., Itou S., Nakajima Y., Tanabe D., Washizuka K., Araki T., Sakurai M., Matsui S., Imamura E., Ueshima K., Yamamoto T., Yamamoto N., Ishikawa H., Nakano K., Unami N., Hamada K., Matsumura Y., Takamura F. (2009) Bioorg Med Chem Lett. 19(16):4679-83.

[43] Seneci P., Bianchi A., Battaglia C., Belvisi L., Bolognesi M., Caprini A., Cossu F., Franco E., Matteo M., Delia D., Drago C., Khaled A., Lecis D., Manzoni L., Marizzoni M., Mastrangelo E., Milani M., Motto I., Moroni E., Potenza D., Rizzo V., Servida F., Turlizzi E., Varrone M., Vasile F., Scolastico C. (2009) Bioorg Med Chem. 17(16):5834-56.

[44] Thompson S.K., Washburn D.G., Frazee J.S., Madauss K.P., Hoang T.H., Lapinski L., Grygielko E.T., Glace L.E., Trizna W., Williams S.P., Duraiswami C., Bray J.D., Laping N.J. (2009) Bioorg Med Chem Lett. 19(16):4777-80.

[45] Tsuji E., Okazaki K., Takeda K. (2009) Biochem Biophys Res Commun. 378(3):494-7.

[46] Nakaishi Y., Bando M., Shimizu H., Watanabe K., Goto F., Tsuge H., Kondo 
K., Komatsu M. (2009) FEBS Lett. 583(1):163-7.

[47] Lund I.K., Andersen H.S., Iversen L.F., Olsen O.H., Moller K.B., Pedersen A.K., Ge Y., Holsworth D.D., Newman M.J., Axe F.U., Moller N.P. (2004) J Biol Chem. 279(23):24226-35.

[48] Xu Y., Liu Y., Lou Z., Qin L., Li X., Bai Z., Pang H., Tien P., Gao G.F., Rao Z.. (2004) J Biol Chem. 279(29):30514-22.

[49] Xu L., Li C., Olson A.J., Wilson I.A. (2004) J Biol Chem. 279(48):50555-65.

[50] Andrews M.J., Mclnnes C., Kontopidis G., Innes L., Cowan A., Plater A., Fischer P.M. (2004) Org Biomol Chem. 2(19):2735-41.

[51] Petrinca A.R., Pietroiusti A., Argentin G., Cicchetti R., Donia D., Gabrieli R., Vignoli I., Magrini A., Divizia M. (2009) Ig Sanita Pubbl. 65(2):169-88.

[52] Dobrovolskaia M.A., Aggarwal P., Hall J.B., McNeil S.E. (2008) Mol Pharm. 5(4):487-95.

[53] Patra C.R., Bhattacharya R., Wang E., Katarya A., Lau J.S., Dutta S., Muders M., Wang S., Buhrow S.A., Safgren S.L., Yaszemski M.J., Reid J.M., Ames M.M., Mukherjee P., Mukhopadhyay D.(2008) Cancer Res. 68 (6):1970-8.

[54] Ljubimova J.Y., Fujita M., Ljubimov A.V., Torchilin V.P., Black K.L., Holler E. (2008) Nanomed. 3(2):247-65.

[55] Chow E.K., Pierstorff E., Cheng G., Ho D. (2008) ACS Nano. 2(1):33-40.

[56] Fujita M., Lee B.S., Khazenzon N.M., Penichet M.L., Wawrowsky K.A., Patil R., Ding H., Holler E., Black K.L., Ljubimova J.Y. (2007) J Control Release. 122(3):356-63.

[57] Rezler E.M., Khan D.R., Lauer-Fields J., Cudic M., Baronas-Lowell D., Fields G.B. (2007) J Am Chem Soc. 129(16):4961-72.

[58] Vinogradov S.V. (2006) Curr Pharm Des. 12(36):4703-12.

[59] Sinha R., Kim G.J., Nie S., Shin D.M. (2006) Mol Cancer Ther. 5(8):1909-17.

[60] Ma B., Nussinov R. (2007) Curr Top Med Chem. 7(10):999-1005.

[61] Churchill E.N., Qvit N., Mochly-Rosen D. (2009) Trends Endocrinol Metab. 20(1):25-33.

[62] Peter M. Fischer (2005) Int J Pept Res Ther. 12(1): 3-19.

[63] Villoutreix B.O., Bastard K., Sperandio O., Fahraeus R., Poyet J.L., Calvo F., Deprez B., Miteva M.A. (2008) Curr Pharm Biotechnol. 9(2):103-22.

[64] Kagan V.E., Bayir A., Bayir H., Stoyanovsky D., Borisenko G.G., Tyurina Y.Y., Wipf P., Atkinson J., Greenberger J.S.,
Chapkin R.S., Belikova N.A. (2009) Mol Nutr Food Res. 53(1):104-14.

[65] Kaler G., Truong D.M., Khandelwal A., Nagle M., Eraly S.A., Swaan P.W., Nigam S.K. (2007) J Biol Chem. 282(33):23841-53.

[66] Truong D.M., Kaler G., Khandelwal A., Swaan P.W., Nigam S.K. (2008) J Biol Chem. 283(13):8654-63.

[67] Segers K., Sperandio O., Sack M., Fischer R., Miteva M.A., Rosing J., Nicolaes G.A., Villoutreix B.O. (2007) Proc Natl Acad Sci U S A. 104(31):12697-702.

[68] Fischer P.M. (2006) Int J Pept Res Ther. 12(1):3-19.

[69] Mulky A., Kappes J.C. (2005) Antimicrob Agents Chemother. 49(9):3762-9.

[70] Mizuno N., Niwa T., Yotsumoto Y., Sugiyama Y. (2003) Pharmacol Rev. 55(3):425-61.

[71] Surajit Bhattacharjya and Ayyalusamy Ramamoorthy (2009) FEBS Journal 6465-6473

[72] Okarvi S.M., Jammaz I.A. (2009) Anticancer Res. 29(4):1399-409.

[73] Rao S., Bockstael K., Nath S., Engelborghs Y., Anne J., Geukens N. (2009) FEBS J. 276(12):3222-34.

[74] Rathinakumar R., Walkenhorst W.F., Wimley W.C. (2009) J Am Chem Soc. 131(22):7609-17.

[75] Ito M, Ito J., Kitazawa H., Shimamura K., Fukami T., Tokita S., Shimokawa K., Yamada K., Kanatani A., Uemura D. (2009) Peptides. 30(6):1074-81.

[76] McVeigh P., Mair G.R., Atkinson L., Ladurner P., Zamanian M., Novozhilova E., Marks N.J., Day T.A., Maule A.G. (2009) Int J Parasitol. 39(11):1243-52.

[77] Sarantseva S.V., Bol'shakova O.I., Timoshenko S.I., Kolobov A.A., Vitek M.P., Shvartsman A.L.(2009) Biomed Khim. 55(1):41-9.

[78] Nakajima E., Goto Y., Sako Y., Murakami H., Suga H. (2009) Chembiochem. 10(7):1186-92.

[79] Lee C.Y., Chen H.H., Lisy O., Swan S., Cannon C., Lieu H.D., Burnett JC Jr. (2009) J Clin Pharmacol. 49(6):668-73.

[80] Orzaez M., Gortat A., Mondragon L., PerezPaya E. (2009) ChemMedChem. 4(2):146-60. 